\title{
Relación entre la cantidad de ARN total hepático y algunos parámetros sanguíneos en la diabetes mellitus experimental
}

\author{
Flores, C.; Márquez, Y.; López-Ortega, A. \\ Unidad de Investigación en Ciencias Funcionales Dr. Haity Moussatché (UNIHM), Facultad de Ciencias \\ Veterinarias, Universidad Centroccidental "Lisandro Alvarado" (UCLA), Barquisimeto, \\ Venezuela, Tel.0251-2592630, E-mail: caf06@hotmail.com.
}

\begin{abstract}
Resumen
Flores, C.; Márquez, Y.; López-Ortega, A.: Relación entre la cantidad de ARN total hepático y algunos parámetros sanguíneos en la diabetes mellitus experimental. Rev. vet. 19: 2, 114-118, 2008. La diabetes mellitus (DM) ha sido asociada con alteraciones de expresión génica en tejidos sensibles a la insulina. El objetivo de este estudio fue determinar la cantidad de ARN total en células hepáticas y su relación con ciertos parámetros sanguíneos vinculados directa o indirectamente con la DM. Se utilizaron ratones adultos NMRI de sexo hembra, distribuidas en tres grupos $(0 ; 15$ y 30 días post-inducción). A los grupos de 15 y 30 días se les administró ip $40 \mathrm{mg} / \mathrm{kg}$ de estreptozotocina durante 5 días. A todos los grupos se les efectuaron determinaciones sanguíneas de glucosa, proteínas totales, triglicéridos y hemoglobina. Semanalmente se determinaron en forma cualitativa $\mathrm{pH}$, glucosa y cuerpos cetónicos en orina. Todos los animales se sacrificaron bajo ligera eterización y en hígado se determinaron ARN total y proteínas totales. Se encontró un aumento significativo en los niveles de glucosa en los animales de 15 y 30 días post-inducción, en comparación al grupo 0 días. El ARN total aumentó significativamente a los 15 días post-inducción, mientras que a los 30 días los animales presentaron una disminución marcada incluso por debajo de los niveles encontrados al día 0 . Al correlacionar el ARN total hepático con los parámetros sanguíneos, se observó que a medida que aumentaban los valores de ARN, se incrementaban los de glucosa $(r=0,60 ; p<0,01)$. Se concluye que en la DM experimental se modifica la expresión génica hepática, cambio que guarda relación con los niveles de glucosa sanguínea.
\end{abstract}

Palabras clave: ratón, diabetes mellitus, glicemia, ARN hepático.

\begin{abstract}
Flores, C.; Márquez, Y.; López-Ortega, A.: Relation between total amount of hepatic RNA and some blood parameters in experimental diabetes mellitus. Rev. vet. 19: 2, 114-118, 2008. Diabetes mellitus (DM) has been associated with alterations of genic sensible weave expression to insulin. The objective of this study was to determine the total amount of RNA in hepatic cells and its relation with some blood parameters. Adult female NMRI mice were used, distributed in three groups $(0 ; 15$ and 30 days post-induction). Streptozotocin (40 $\mathrm{mg} / \mathrm{kg} \mathrm{ip}$ ) was administered during 5 days to the group of 15 and 30 days. In blood of all groups it was determined glucose, total protein, triglycerides, and hemoglobin. In addition, $\mathrm{pH}$, glucose and ketonic bodies were qualitatively determined in urine. All animals were sacrificed under light etherisation, and in liver it was determined total RNA and total protein. It was observed a significant increase in glucose blood level in animals of 15 and 30 days post-induction, in comparison to 0 day group. Total RNA significantly increased 15 days post-induction in comparison to group 0 day, whereas the 30 days group showed a noticeable diminution below the levels found for the 0 days group. When correlating the hepatic total RNA with blood parameters, it was observed that both RNA values and glucose levels increased at the same time. It is concluded that in experimental DM, the hepatic genic expression is modified and this change is linked to blood glucose levels.
\end{abstract}

Key words: mice, diabetes mellitus, glycemia, hepatic RNA. 


\section{INTRODUCCIÓN}

La diabetes mellitus (DM) es el trastorno metabólico más frecuente del páncreas endocrino en perros. Resulta de la deficiencia relativa o absoluta de insulina debido a la secreción deficiente de las células beta del páncreas ${ }^{4}$.

Algunos investigadores señalan que la expresión génica de los tejidos sensibles a la insulina se altera cuando la señal hormonal está ausente en forma total o parcial; además han revelado que el ARN total de las células sanguíneas está disminuido en diabéticos ${ }^{1,9}$ y que las alteraciones génicas son las responsables de las complicaciones que se observan en la $\mathrm{DM}^{3}$. De igual modo se reporta que los cambios metabólicos observados en la DM (aumentos de glucosa, triglicéridos, colesterol, entre otros), tienen un efecto directo sobre el gen de la superóxido dismutasa en embriones de ratas y quizás en los genes de otras enzimas antioxidantes ${ }^{16,20}$.

Se han identificado varios nuevos genes que son regulados positivamente en riñones de diabéticos, un ejemplo de ello es el factor transformante beta, el cual promueve la secreción de proteínas, tales como el factor de crecimiento tisular, cuya expresión se correlaciona con los cambios en los tejidos observados en la nefropatía diabética de la población adulta ${ }^{17}$.

El aumento en la actividad gluconeogénica puede ser responsable de la elevación plasmática de glucosa en la DM tipo 2, lo cual está dado por el aumento de los glucocorticoides intracelulares y el AMPc, debido a que contribuyen a la activación de vías aberrantes de señalización para la producción de glucosa, durante la inducción de la expresión de genes de enzimas gluconeogénicas ${ }^{8}$. Asimismo, se afirma que la fosfopiruvato carboxikinasa (enzima encargada de controlar la tasa de gluconeogénesis), se encuentra alterada en individuos diabéticos, lo que hace suponer que hay una alta producción de glucosa por parte del hígado y muy probablemente sea la responsable del mantenimiento de la hiperglicemia en estos pacientes ${ }^{6,19}$.

Tanto la proteína 3-hidroxi-3-metilglutaril-CoA reductasa hepática como el ARNm están sustancialmente reducidos en animales diabéticos, a consecuencia de la disminución en la trascripción y son rápidamente restaurados por la administración de insulina ${ }^{10}$. El hígado es uno de los órganos más afectados en la DM, a pesar de ello muy pocos estudios se han realizado para determinar posibles alteraciones en la formación de ARN total hepático y su relación con los cambios observados en algunos parámetros sanguíneos como hemoglobina, glucosa, triglicéridos, proteínas totales y albúmina, entre otros.

El objetivo de este estudio fue determinar la cantidad total de ARN hepático en roedores con DM experimental y verificar su eventual relación con ciertos parámetros sanguíneos relacionados directa o indirectamente con la endocrinopatía. El presente trabajo es parte de un proyecto que propone profundizar el conocimiento de la expresión de genes que codifican algu- nas enzimas participantes del metabolismo hepático en este modelo experimental.

\section{MATERIAL Y MÉTODOS}

Muestra. Las unidades experimentales utilizadas fueron ratones hembras de la cepa NMRI no consanguíneos, adultos de $30 \mathrm{~g}$ de peso promedio. Aleatoriamente se conformaron tres grupos $(0 ; 15$ y 30 días post-inducción de DM), con 40 animales por grupo. Todos los ejemplares permanecieron bajo ciclos de luz de 12 horas. Fueron alimentados con un concentrado comercial para roedores y dispusieron de agua filtrada ad libitum. Diariamente se efectuaron mediciones del agua y del alimento consumido por cada uno de los grupos. La temperatura promedio anual en el bioterio utilizado es de $27,5 \pm 0,44^{\circ} \mathrm{C}$ y la humedad relativa del $65 \%{ }^{7}$.

Inducción de diabetes mellitus. Se realizó mediante la administración de una inyección intraperitoneal diaria de estreptozotocina (STZ) en buffer citrato de sodio $0,05 \mathrm{M} \mathrm{pH} 4,5$ a la dosis de $40 \mathrm{mg} / \mathrm{kg}$ de peso corporal durante cinco días de acuerdo al clásico modelo propuesto por Like y Rossini ${ }^{12}$.

Determinación de parámetros sanguíneos. A los 0 ; 15 y 30 días post-tratamiento, sin someter a los animales a ayuno previo, se les extrajo sangre mediante punción de la vena coccígea media, sin anticoagulante. Con el suero obtenido se determinaron por espectrofotometría, mediante kits comerciales, las concentraciones de triglicéridos (con patrón de $200 \mathrm{mg} / \mathrm{dl}$ ), proteínas totales $(4,9 \mathrm{~g} / \mathrm{dl})$, albúmina $(3,0 \mathrm{~g} / \mathrm{dl})$ y glucosa $(100 \mathrm{mg} /$ dl). El valor de hemoglobina se obtuvo por técnica de química seca. Semanalmente se extrajeron muestras de orina, efectuándose un urianálisis cualitativo mediante tiras reactivas (Combur, Roche).

Cuantificación de la concentración de ARN total en hígado. A los 0; 15 y 30 días post-inducción de DM, los roedores fueron sacrificados bajo ligera eterización, tras lo cual se les disecó el hígado y se separó una mues-

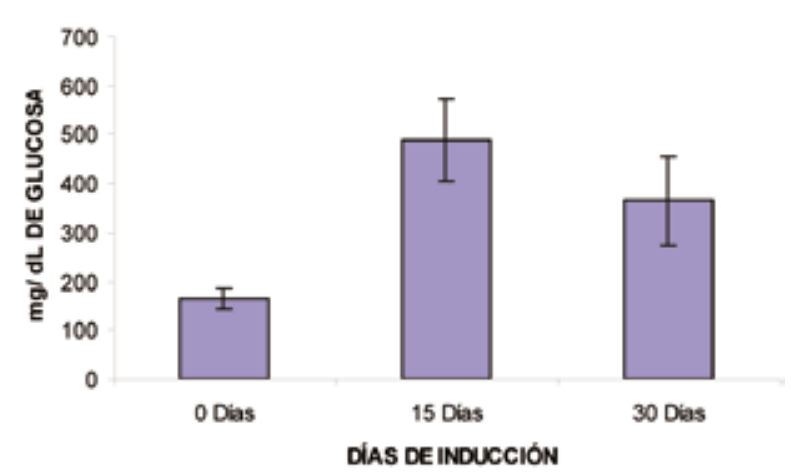

Figura 1. Concentración de glucosa sanguínea en ratones con diabetes mellitus inducida por estreptozotocina. ${ }^{*} \mathrm{p}<0,05$ en relación al grupo 0 días. 
tra que se colocó en tubos estériles, almacenándose a $-70^{\circ} \mathrm{C}$. El ARN total se determinó mediante el kit RNeasy Project (Qiagen, USA). Se homogenizaron $20 \mathrm{mg}$ de la muestra de hígado (Pellet pestle Motor Kontes) en $350 \mu \mathrm{l}$ de buffer de lisis, se centrifugó el homogenizado durante 2 minutos a $15.000 \mathrm{x}$ g en microcentrífuga Eppendorf 5402 y el sobrenadante fue colocado en la columna del kit y lavado con diferentes buffers del kit. La cantidad de ARN total fue calculada según la metodología del kit RNeasy Project y se expresó en $\mu \mathrm{g}$ de ARN/mg de proteína.

Concentración de proteínas hepáticas. Otra porción de hígado se pesó y se diluyó 1:3 en buffer tris sacarosa pH 7,4 tras lo cual se homogenizó en un Euroturrax T20 de $27.000 \mathrm{rpm}$ (Labortechnik, Alemania). El homogenizado se mantuvo en hielo y en el sobrenadante se determinaron proteínas totales por kit Pierce (Rockford, USA), con un patrón de albúmina sérica bovina $(2 \mathrm{mg} / \mathrm{ml})$.

Estudio histopatológico. Para evidenciar células de los islotes pancreáticos y grado de infiltración grasa a nivel hepático, se realizaron cortes de páncreas e hígado, se fijaron en formol al 10\% y se colorearon con hematoxilina-eosina (H\&E).

Análisis estadístico. Los resultados de la química sanguínea obtenidos en los diversos experimentos se analizaron estadísticamente mediante ANOVA a una vía, con significancia de 95\% $(\mathrm{p}<0,05)$. Para determinar el grado de asociación entre la cantidad de ARN total presente en las células hepáticas con el resto de las variables se utilizó correlación de Pearson con significancia del 99\% $(\mathrm{p}<0,01)$.

\section{RESULTADOS}

Características conductuales de los animales experimentales. En el estudio del comportamiento de las unidades experimentales se evidenció un aumento en el consumo de alimento (polifagia) y agua (polidipsia), dos de los signos clásicos de DM. Los roedores también se tornaron agresivos. Todas estas condiciones aumentaron al avanzar los días post-inducción.

Análisis cualitativo de parámetros en orina. Se pusieron en
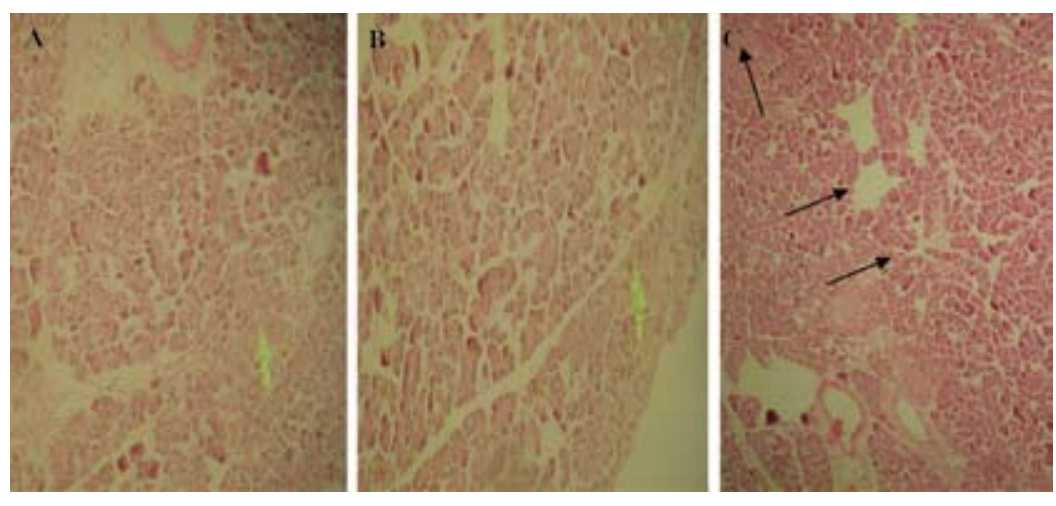

Figura 3. A: páncreas de ratón con diabetes mellitus de 15 días de evolución. B: a los 30 días post-inducción experimental. C: control sano (día 0). Flechas indican islotes de Langerhans (H\&E, 40X).
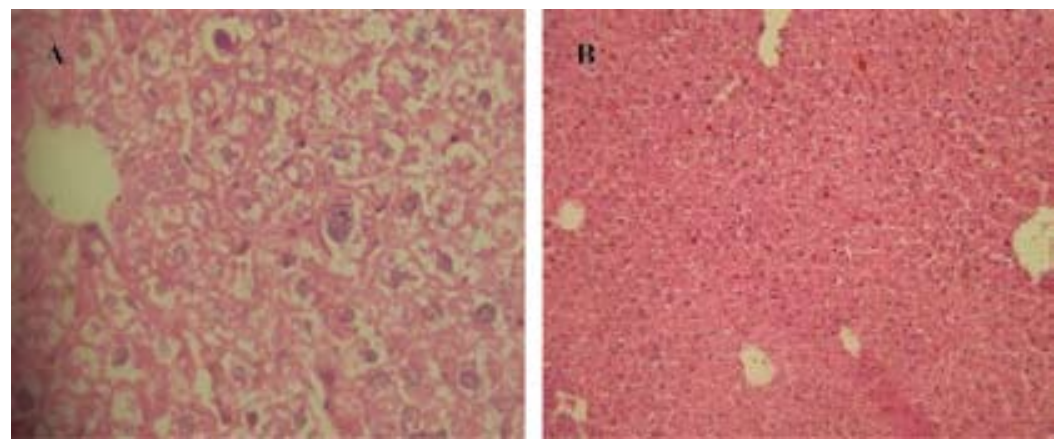

Figura 4. A: hígado de ratón a los 30 días post-inducción de diabetes mellitus, que revela ligera infiltración grasa (H\&E, 60X). B: hígado de ratón a los 0 días post-inducción (H\&E, 40X). 
evidencia altos niveles de glucosa, entre 100 y 1000 $\mathrm{mg} / \mathrm{dl}$, tanto a los 15 como a los 30 días post-inducción. También se revelaron cuerpos cetónicos, variando entre 1 y 3 cruces en ambos grupos ( 15 y 30 días post-inducción). A los 0 días, no se registró presencia de glucosa ni cetonas en las muestras de orina. Estos hallazgos indicaron el establecimiento de un cuadro diabético en los grupos experimentales.

Determinación de parámetros sanguíneos. $\mathrm{La}$ glicemia aumentó significativamente $(\mathrm{p}<0,05)$ hasta el final de la experiencia. Se pudo observar que a los 15 días post-inducción, el nivel de glucosa triplicó el valor obtenido al día 0 (Figura 1). En cuanto a los niveles de triglicéridos séricos, éstos tendieron a aumentar a medida que avanzaron los días de inducción de DM (Tabla 1) pero sin alcanzar significancia estadística. Los valores obtenidos para hemoglobina, proteínas totales y albúmina no presentaron diferencias significativas entre los grupos.

Concentración total de ARN hepático. Los niveles de ARN total en hígado presentaron una elevación significativa $(\mathrm{p}<0,05)$ a los 15 días post-inducción, mientras que a los 30 días los valores tendieron a disminuir aunque de manera no significativa (Figura 2).

Correlación entre parámetros sanguíneos y niveles de ARN total en hígado. Los niveles de ARN total hepático presentaron una correlación directa muy significativa $(\mathrm{r}=0,60 ; \mathrm{p}<0,01)$ con las concentraciones sanguíneas de glucosa.

Cambios histopatológicos. En el páncreas se registró una marcada disminución y deterioro de los islotes de Langerhans en los animales a los 15 y 30 días post-inducción de DM (Figura 3A y 3B) al ser comparados con los animales no diabéticos, es decir, a los 0 días post-inducción (Figura 3C). Ello demuestra el efecto deletéreo de la STZ sobre los islotes de Langerhans. Mientras que los controles no acusaron cambios, los hígados de ratones diabéticos revelaron grados variables de degeneración grasa, como muestra la Figura 4.

\section{DISCUSIÓN}

La DM es una patología de importancia epidemiológica; proyecciones realizadas por la Sociedad Venezolana de Endocrinología y Metabolismo demuestran el avance de la enfermedad a escala mundial para los años 1995, 2000 y 2025 y se reporta una prevalencia de $4,2 \%$, con una proporción según el sexo, cercana a 12 mujeres por cada 10 hombres, la mayoría de los casos en pacientes humanos mayores de 20 años ${ }^{15}$. En perros representa el trastorno metabólico más frecuente del páncreas endocrino ${ }^{4}$.

La STZ induce DM experimental al dañar las células beta del páncreas, suprimiendo la síntesis de insuli- na. En el presente estudio se observó que los animales con DM inducida con STZ, presentaron una marcada hiperglicemia que se mantuvo hasta el final de la experiencia (30 días) con los más altos niveles de glucosa a los 15 días post-inducción. Estos resultados coinciden con estudios realizados por diferentes autores, los cuales reportan elevados niveles de glucosa sanguínea luego de la inyección de STZ bajo el protocolo de Like y Rosssini ${ }^{5,11,13}$.

El efecto diabetógeno de la STZ se debe a su capacidad de destrucción de los islotes de Langerhans, hallazgo corroborado en este estudio al realizar cortes histológicos del páncreas, en los cuales se observó una marcada disminución y deterioro de los islotes en los animales de 15 y 30 días post-inducción, al compararlos con los animales no diabéticos, lo que indicaría que la DM experimental inducida con STZ es de tipo 1, la cual se caracteriza por presentar deficiencia de la producción de insulina por destrucción de los islote de Langerhans ${ }^{12}$.

Los niveles de proteínas totales, albúmina, hemoglobina y triglicéridos no mostraron diferencias significativas entre los grupos; en relación a este último, se ha señalado que en pacientes diabéticos no tratados con insulina se pueden observar niveles normales de triglicéridos ${ }^{14}$. Los niveles de ARN registraron una correlación directa muy significativa con las concentraciones de glucosa sanguínea. De acuerdo a estos resultados se puede inferir que las alteraciones génicas en el tejido hepático de los animales diabéticos pudieron haber contribuido a la elevación de la glucosa presentada por estos animales. Cabe señalar que el hígado es productor de glucosa a través de la gluconeogénesis en los casos donde existe deficiencia de insulina.

Se afirma que los principales parámetros que afectan la salida de glucosa hepática son la concentración o disponibilidad de sustratos y la actividad de enzimas reguladoras como fosfoenolpiruvatokinasa y glucosa6-fosfatasa, ambas reguladas por mecanismos transcripcionales y no transcripcionales, además de una tercera llave enzimática, la fructosa-1,6-bifosfatasa, también regulada por inhibición competitiva a través de la fructosa 2,6-bifosfato ${ }^{2}$.

Recientemente se ha señalado que para que exista el efecto supresor de la gluconeogénesis por la insulina $^{8}$, debe existir un perfecto balance en el mecanismo de señalización celular llevada a cabo por el sistema tiroxincinasa ${ }^{12}$. La insulina es la hormona más importante para inhibir la gluconeogénesis, debido a que suprime la expresión de genes que son la clave para la formación de enzimas gluconeogénicas; además, en los pacientes diabéticos la actividad de dichas enzimas está alterada por la deficiencia de insulina, lo que contribuye a la salida de glucosa de origen hepático y a la elevación los niveles de glucosa en sangre ${ }^{18}$.

En conclusión, los animales con DM experimental inducida con STZ presentan elevación de los niveles sanguíneos de glucosa, con acmés a los 15 días postinducción. Se establece una correlación directa signi- 
ficativa entre las concentraciones de glucosa y la cantidad de ARN total hepático, hecho que se atribuye a una alteración en la expresión génica a nivel de tejido hepático, propia de la DM experimental inducida con STZ.

\section{REFERENCIAS}

1. Adan C, Arabujo L. 2002. Alteración de los patrones polipeptídicos en leucocitos de pacientes con diabetes mellitus. Acta Cient Venez 53: 50.

2. Barthel A, Schmoll D. 2003. Novel concepts in insulin regulation of hepatic gluconeogenesis. Am J Phys Endocr Metab 285: E685-E692.

3. Ducluzeau P, Perretti N, Laville M, Andrelli F, Vega N, Riou J, Vidal H. 2001. Regulation by insulin of gene expression in human skeletal muscle and adipose tissue. Diabetes 50: 1134-1142.

4. Feldman E, Nelson R. 2000. Endocrinología de perros y gatos, McGraw-Hill, México, p. 370-390.

5. Fisher SJ, Kahn CR. 2003. Insulin signaling is required for insulin's direct and indirect action on hepatic glucose production. J Clin Invest 111: 463-468.

6. Gómez-Valadés AG, Vidal-Alabró A, Molas M, Boada J, Bermúdez J, Bartrons R, Perales JC. 2006. Overcoming diabetes-induced hyperglycemia through inhibition of hepatic phosphoenolpyruvate carboxykinase (GTP) with RNAi. Mol Ther 13: 401-410.

7. González M. 2003. Efecto del extracto de hojas de nin (Azadirachta indica) sobre la úlcera gástrica inducida en ratas. Trabajo de Ascenso, Universidad Centroocidental Lisandro Alvarado, Cabudare (Venezuela) p. 11.

8. Krones-Herzig A, Mesaros A, Metzger D, Ziegler A, Lemke U, Bruning JC, Herzig S. 2006. Signal-dependent control of gluconeogenic key enzyme genes through coactivator-associated arginine methyltransferase. $\mathrm{J} \mathrm{Biol}$ Chem 281: 3025-3029.

9. Keyloun N, Bermudez G. 2002. El ARN celular total en leucocitos como indicador de la expresión genética en pacientes con diabetes mellitus. Acta Cient Venez 53: 50-51.

10. Lagor WR, de Groh ED, Ness GC. 2005. Diabetes alters the occupancy of the hepatic 3-hydroxy-3-methylglu-
taryl-CoA reductase promoter. J Biol Chem 280: $36601-$ 36608 .

11. Lee JS. 2006. Effects of soy protein and genistein on blood glucose, antioxidant enzyme activities, and lipid profile in streptozotocin-induced diabetic rats. Life Sci 79: 15781584.

12. Like AA, Rosssini AA. 1976. Streptozotocin-induced pancreatic insulitis new model of diabetes mellitus. Science 193: 415-417.

13. López-Ortega A. 1997. Acción de diferentes antioxidantes sobre la modificación de la LDL humana causada por las células endoteliales y por el ión cúprico. Trabajo de Ascenso, Universidad Centroccidental Lisandro Alvarado, Cabudare (Venezuela), p. 20-22.

14. Mendoza C. 2004. Estrés oxidativo hepático en ratones hembras hasta los 45 días post-inducción de diabetes mellitus por estreptozotocina. Trabajo de Ascenso, Universidad Centrocidental Lisandro Alvarado, Cabudare (Venezuela), p. 32-35.

15. Nakai T, Yamada S, Tamai T, Kobayashi T, Hayashi T, Takeda R. 1979. The effects of streptozotocin diabetes on hepatic triglyceride lipase activity in the rat. Metabolism 28: $30-39$.

16. Rigalleau V, Baillet-Blanco L, Perlemoine C, Salmi LR, Gin H. 2005. Triglycerides high in serum are associated with increased need for insulin requirement in poorly controlled type 2 diabetic patients. Diab Med 22: 877-881.

17. Sociedad Venezolana de Endocrinología y Metabolismo. 2003. Publ. Consejo Nacional de Diabetes Tipo 2, Caracas (Venezuela), p. 9-10.

18. Wallner EI, Wada J, Lin S, Pan X, Renddy J, Cugh S, Kanwar Y. 2002. Renal gene expression in embryonic and newborn diabetic mice. Exp Nephrol 10: 130-138.

19. Xu H, Yang Q, Shen M, Huang X, Dembski M, Gimeno R, Tartaglia L, Kapeller R, Wu Z. 2005. Dual specificity MAPK posphatase 3 activates PEPCK gene transcription and increases gluconeogenesis in rat hepatoma cells. $J$ Biol Chem 280: 36013-36018.

20. Zaken V, Kohen R, Ornoy A. 2001. Vitamins C and E improve rat embryonic antioxidant defense mechanism in diabetic culture medium. Teratology 64: 33-44. 